\title{
TOWARDS AN EFFICIENT INTEGRATION, STRUCTURE AND EXPLORATION OF LANDSCAPE ARCHITECTURE PROJECT INFORMATION
}

\author{
Franck FAVETTA ${ }^{+}$, Robert LAURINI* \\ Research Center for Images and Intelligent Information Systems ${ }^{*}$, INSA-Lyon F-69622 Villeurbanne \\ National School of Higher Studies in Landscape Architecture and Nature ${ }^{+}$, cs2902 F-41029 Blois cedex \\ franck.favetta@free.fr, robert.laurini@liris.cnrs.fr
}

\begin{abstract}
Landscape Architecture projects have many specific requirements such as particular multimedia and geographic data integration and structure, information preview, userfriendly interface, and means of multi-actor participation. This article presents a solution for an efficient, quick and user-friendly integration, structure, exploration and management of landscape information. Our proposal extends different existing solutions and introduces useful preview abilities. A recently developed prototype implements the solution.

Key-Words: multimedia, interactive and visual interfaces, metaphors, GIS, landscape architecture, sound landscapes
\end{abstract}

\section{INTRODUCTION}

The objective of Ecole Nationale Supérieure de la Nature et du Paysage (National School of Higher Studies in Landscape Architecture and Nature) is to develop research in the field of multimedia and Geographic Information Systems [1] in order to meet Landscape Architecture project requirements. This article presents a novel approach in this area. Our motivation for this work initially comes from many discussions with landscape architects and students.

The next section details our motivation by describing what a landscape project consists of and presents its requirements. The third section deals with useful research background. The fourth section presents our solution. The fifth section introduces the prototype we have developed. The last section concludes and presents perspectives.

\section{LANDSCAPE PROJECT AND REQUIREMENTS}

We can summarize Landscape Architecture projects through five stages: site analysis, solution proposal and design, proposal communication to other actors, realization on the site, and maintenance.

As a first step, this article essentially focuses on the analysis stage, especially on the structure and visualization requirements of Landscape Architecture projects.

During the analysis phase, in order to formulate a diagnosis, the landscape architect first collects and measures a sufficient amount of information on site, such as natural information (e.g., vegetation, mineralogy, geology, hydrology), infrastructure information (e.g., cadaster, buildings, networks, architecture), and social and economic information (e.g., census, economic and geo-political factors and actors, resources, site history). To achieve this goal, the landscape architect collects information from all available sources such as the general public, local governments, the media, libraries, and the Internet. He also performs measures himself on the site, especially pictures, videos, notes, sketches, and distance measures. The landscape architect essentially handles geographical information (maps, 3D maps) and multimedia information such as images, videos, texts, and 3D models. Moreover, information is usually visual, but also presents an important audio dimension (landscape map of sounds often called soundscape, voice recordings) and temporal dimension (moving viewers in a car or on a train, walkers, animated or moving landscapes).

As visiting time on the site is usually short, the architect usually takes a maximum amount of information for later analysis away from the site. The amount of information is generally very large, unorganized, and sometimes unnecessary. It needs to be filtered and structured. Maps are stored as GIS data or as images, and handled through GIS interfaces or image software applications. Multimedia files are stored on the computer file system, divided in folders, and edited through related software editors. Collected documents are geographically linked: pictures are located at precisely defined points of view and pictures are oriented in specific directions, videos and sounds are recorded in particular places, text documents and images can concern one special object in the landscape, several maps can concern several neighboring places, and so on. Information storage and access do not fit landscape information structure. The architect must make this correspondence himself. Landscape project information strongly calls for solutions to be structured and manipulated in an efficient way. 
Furthermore, architects need to view the existing site and also to preview the result of their proposals. They need a sufficient, not perfect, realism, and they also need to consider a certain sensitive aspect. Systems must be userfriendly and allow architects to quickly acquire a sufficient representation of the existing site and to quickly preview different project proposals.

Other requirements exist such as spatial and economic calculation means, project evolution and versions management. An important requirement concerns management and communication among many project actors such as other architects and designers on the team, customers, local authorities, citizens, economic actors, and contractors.

\section{USEFUL BACKGROUND}

Existing systems present notable features for our field of interest [1]. This section shortly describes them.

3D landscape visualization systems [2] offer the advantage of exploring existing sites and previewing proposal results. Many GIS 3D extensions exist [3] as well as non-GIS-based systems. Online solutions also exist such as Geo-VRML (http://www.geovrml.org). Augmented Reality is also interesting by adding virtual objects to the real world [4]. 3D technologies are more and more widespread but require 3D data acquisition. In the case of Landscape Architecture, the architect usually has to model the existing site and the project result in 3D. This commonly takes a long time, and architects find it quicker to touch up pictures. 3D offers realism, but realism requires expert skills and time to be achieved. Landscape architects also dislike 3D landscape images for their numerical insensitive aspect.

Three other systems are interesting for the structure they propose and their interface handling. Hypermaps [5] link maps by geographically placing links on a map to other maps. Argumaps [6] geographically locate information by placing metaphorical pins on maps and attaching information to them. This intuitive metaphor can be used to manage discussions between different users who can exchange arguments on the map. It can also allow users to vote on different proposals and can become a base for Public Participative project communication. Direct Manipulation [7] recommends to always keep the focus on objects of interest and to directly act on objects instead of using intermediate commands.

Three other systems from the industry also present interesting features. In the MappyVisiocity system (http://www.mappyvisiocity.com), the user can explore a street by passing from a front door image to another, following a street map. With widely used 360-degree panorama technologies such as Apple's QuickTimeVR (http://www. apple.com/quicktime/technologies/qtvr), the user can virtually visit a place by turning and zooming on a 360-degree picture around a particular point. In the Datria system (http://www.datria.com/demo.php), a moving person remotely records voice reports that are geographically located thanks to a Global Positioning System and wireless phone. Companies use it to manage geographically distributed employees.

\section{PROPOSAL}

In this section, we propose a solution that takes advantage of and extends previously presented systems with new features. We propose a suited way to structure Landscape Architecture project information. We also introduce visualization features that fit the landscape architect's concerns such as useful preview means.

\subsection{Structure and multimedia integration}

We propose to structure landscape architecture data by adding a meta-data level into the existing operating system file hierarchy and GIS database. Meta-data are in part composed of geographical links. In our system, any map document can potentially contain links geographically located on it. Maps can be GIS maps as well as image documents in the file system. In this paper, we focus on image document map management. Links are connected to other maps and also to various multimedia documents in the file system: texts, videos, sounds, video slides, spreadsheets, graphs, PDF files. The location of links on the map corresponds to the geographical location of documents on the real site. As we find location information insufficient, the system also stores other geographical meta-data like the orientation of pictures that the architect takes on the real site and the trajectory of video recordings that he makes.

The architect himself provides meta-data information through our system interface. He sets link locations on maps. He chooses image document maps and other linked multimedia documents in the file system. He also defines picture orientations and movie trajectories. We can improve this meta-data collection by automating some part of it. Picture locations can be automatically captured on the real site thanks to a Global Positioning System, and picture orientations can be measured thanks to an electronic compass. As a first step, our system manages hand-collected meta-data provided by the architect.

\subsection{Visualization of landscape information}

In our interface (Figure 1), spatial meta-data are graphically represented. Icons are used to represent multimedia documents located at precisely defined points. Polygons are used to indicate documents that concern a geographical area. Lines indicate map sections. Arrows represent picture orientations, video trajectories and section orientations. By viewing an icon, the architect can understand the type of a linked document (text, video, another image, video slides, 


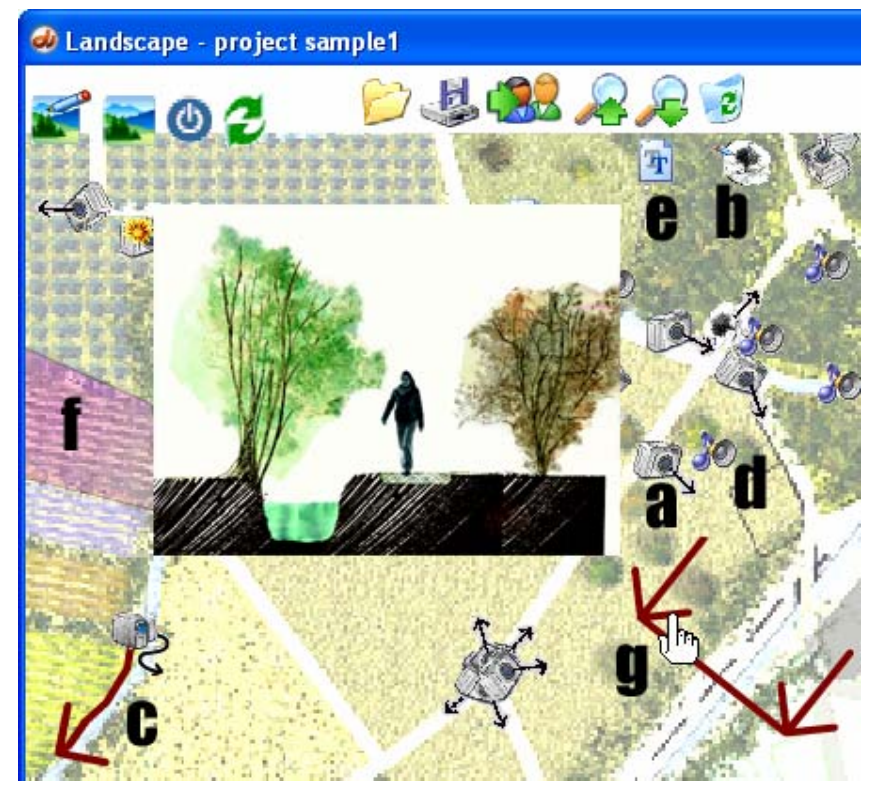

Figure 1. (a) Oriented picture:<smiles>[C]1C=C[C]2C=C[C]1C2</smiles>

(b) sketch:

(c) Video: 2 and its recording trajectory (d) Sound: 8 (e) Text: T (f) area linked to a document

(g) Map section. As the cursor moves over the section, the linked image document is previewed above

spreadsheets, graphs, PDF files). The user can also personalize linked document type with specific icons, e.g., map, picture or sketch icons can all represent an image document. As introduced before, the architect himself defines spatial meta-data. He freely places the pinned icons on the map. He chooses the orientation of pictures. He also draws areas, sections lines, and arrows on the map. At any time, he can modify the location and the orientation of graphical elements. The architect chooses in the file system the multimedia files that become the linked documents.

The architect uses icons and other graphical elements to navigate from an image document to another. He can define new graphical links on any other image document. The architect also uses graphical links to edit a multimedia document through an external software editor. Our system does not replace the usual software edition of images and other multimedia documents. It offers an additional suited structure and global visualization, and provides full interoperability with usual software edition.

\subsection{Landscape information preview and virtual walker}

To improve visualization abilities, our system reacts when the mouse passes over the icons by directly previewing documents: images are viewed, videos are played, first pages of texts are shown, and sounds are played.

We also introduce a virtual walker ( $v w)$ metaphor that offers a way to simulate a person's movement on an image document map. The architect can convert the interface to a

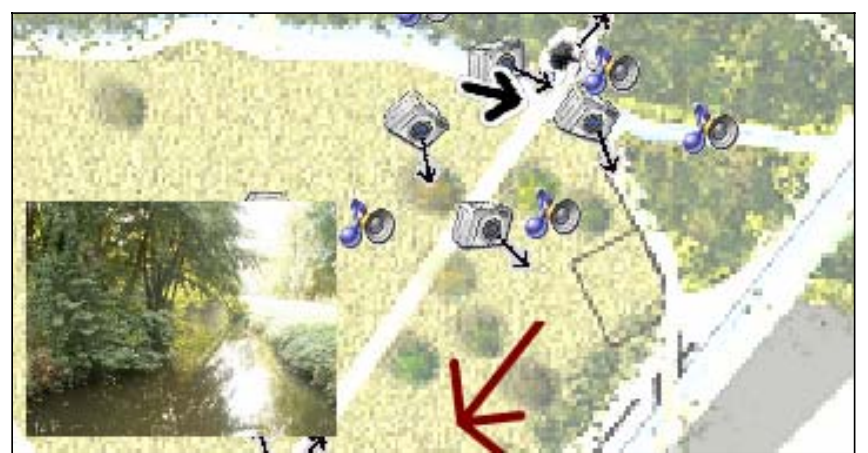

Figure 2. Virtual walker mode and preview

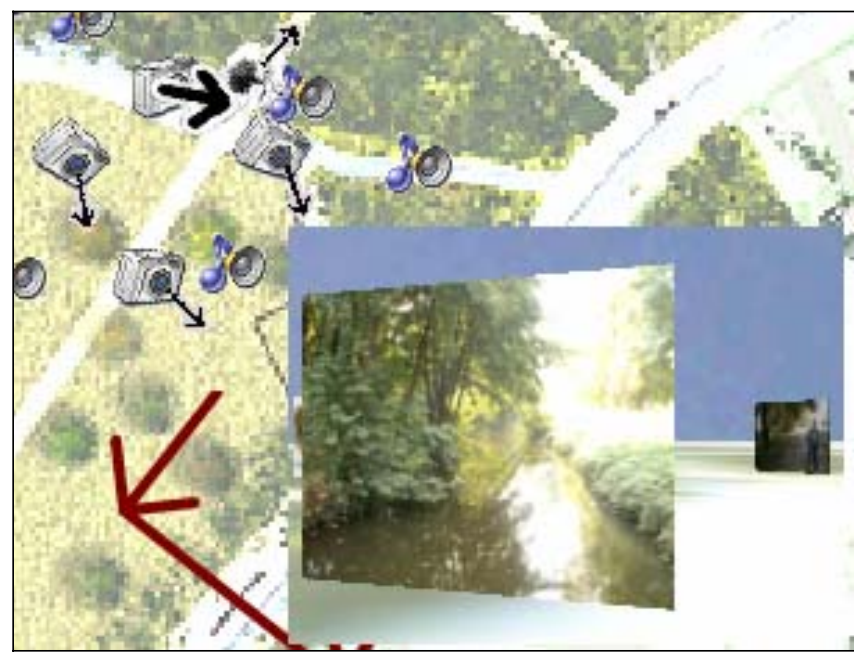

Figure 3. Virtual walker 3D mode

$v w$ mode. In this mode, the mouse becomes the $v w$. An arrow indicates the direction of movement. The $v w$ reacts with linked multimedia documents when approaching them on the map. The interface previews the close images and videos. The distance from the documents affects the preview. The longer the distance, the smaller the preview size is. Moreover, as the $v w$ mode's goal is to simulate real movement, only oriented pictures and videos are previewed and they are displayed only if the $v w$ direction approximately matches their orientations. In order to simulate the soundscape (sound landscape), the $v w$ also hears every sound around it when approaching related sound icons. The closer the sound icon, the louder the sound is. Several surrounding sounds are mixed. This feature adds a real sound map dimension to usual visual maps.

To improve preview capability, the system also offers a $v w 3 D$ mode (Figure 3 ). In 3D mode, the map becomes the floor and the $v w$ can explore it by moving across it. In this virtual preview, oriented images are mapped on vertical planes and are still only visible in the correct image orientation. 3D scene is automatically generated thanks to meta-data. The system automatically maps linked images on vertical planes as textures. It also rotates the planes to the right image orientations. 


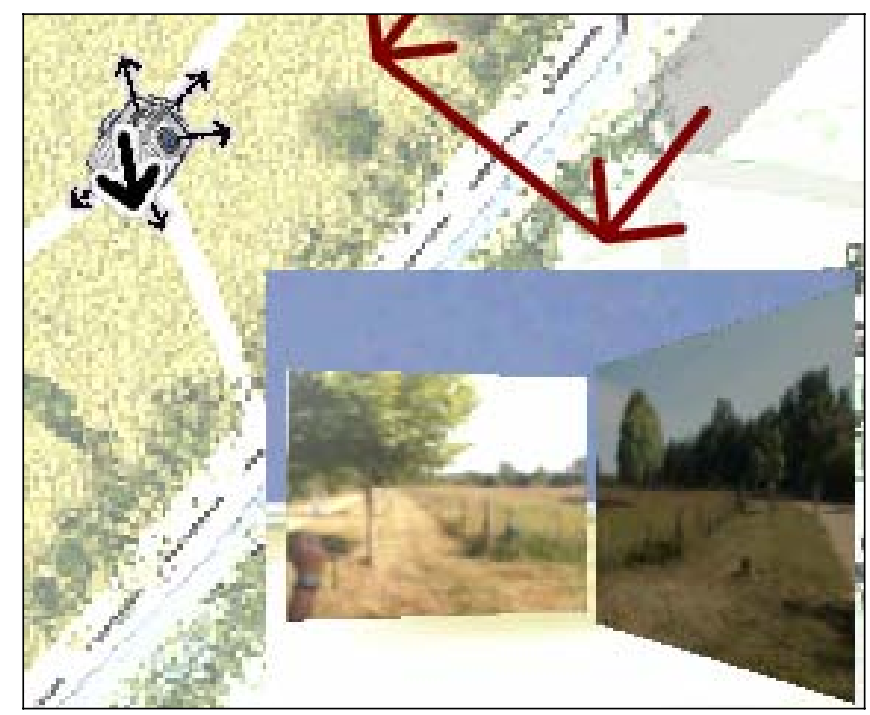

Figure 4: A 360-degree panorama in the virtual walker $3 D$ mode

The $v w$ 3D mode can be seen related to 3D GIS visualization systems like [8], but instead of 3D models, the $v w$ 3D objects are textured 3D planes, automatically generated, that do not require 3D modeling time by the user.

$v w 3 \mathrm{D}$ mode extends the file system visualization to three dimensions like TDFSB (http://www.determinate.net/ webdata/seg/tdfsb.html) and like 3D operating systems interfaces such as Sun's Project Looking Glass (http://www.sun.com/software/looking glass). In these interfaces, spatial information, such as the location of files, does not express any particular semantics. In $v w$ mode, spatial information on the interface expresses geographical information in the real site.

The $v w$ 3D mode allows 360-degree panoramas (Figure 4): we can place pictures in a circle configuration around any chosen spot on the map. Realism can be lower than with classic 360-degree panorama technologies, but the solution is quite easy to realize and the $v w$ can explore the photographed area beyond one unique spot.

\section{PROTOTYPE}

A recently developed prototype integrates all of the functionalities presented in the previous section. Illustrations of the previous section are prototype screenshots. The prototype, called Landscape, is in Beta version. It is available on both Windows and MacOsX operating systems. It is downloadable with a landscape project example at its website location (http://franck .favetta.free.fr/landscape). Project files respect an XML format for better interoperability with future programs.

As animation, interactivity, sound and video constitute a major part of our solution, the use of the prototype is recommended to fully discover our proposal.

\section{CONCLUSIONS AND FUTURE WORK}

We introduced and implemented a novel approach that permits an efficient, quick and user-friendly structure and management of landscape information suited to Landscape Architecture project requirements. It fully integrates a temporal dimension and multimedia information such as video and sound. It also offers useful preview features. We developed a prototype to implement these abilities.

We are working on the creation of tests to check the accuracy of the prototype for landscape architects' needs. We plan to submit tests to Landscape Architecture students and professionals.

As a first step, our work focuses on the analysis phase of Landscape Architecture projects. Our proposal also presents potential abilities for the project design and communication phases. An important future step is to integrate the communication and participation of the numerous landscape project actors.

\section{REFERENCES}

[1] R. Laurini, "Information Systems for Urban Planning: A Hypermedia Cooperative Approach", Taylor and Francis, London, New-York, 308 p., February 2001.

[2] S. M. Ervin, H. H. Hasbrouck, "Digital Techniques for Landscape Visualization", McGraw-Hill Professional Publishing, 2001.

[3] U.S. Army, "Survey of Terrain Visualization Software", U.S. Army Topographic Engineering Center, Topography, Imagery and Geospatial Research Division, Data Representation Branch, 28 November 2005. http://www.tec.army.mil/TD/tvd/survey

[4] D. Schmalstieg, A. Fuhrmann, G. Hesina, Zs. Szalavari, L. M. Encarnação, M. Gervautz, W. Purgathofer, "The Studierstube Augmented Reality Project", Presence - Teleoperators and Virtual Environments, MIT Press, vol.11(1), February 2002. http://www.studierstube.org/research_master.php

[5] R. Laurini, D. Thompson, "Fundamentals of Spatial Information Systems", Academic Press, A.P.I.C. Series No 37, Hardcover, March 1993.

[6] C. Rinner, "Argumentation maps: GIS-based discussion support for on-line planning", Environment and Planning B: Planning and Design, vol.28(6), pp.847-863, 2001.

[7] B. Schneiderman, "Direct manipulation: A step beyond programming languages", IEEE Computer, vol.16, pp. 57-69, 1983.

[8] G. Faber, H. Whyte, "Multi-Faceted Community Planning: Impacts, Forecasts, and 3D Visualization", Urban and Regional Information Systems Association Annual meeting, Montego Bay, Jamaica, 2001 